\title{
Leitthema
}

Bundesgesundheitsbl 2016 $\cdot 59: 153-165$

DOI 10.1007/s00103-015-2275-6

Online publiziert: 2. Dezember 2015

(C) Springer-Verlag Berlin Heidelberg 2015

CrossMark

\author{
Thomas Lampert ${ }^{1} \cdot$ Matthias Richter $^{3} \cdot$ Sven Schneider ${ }^{5}$ Jacob Spallek ${ }^{2}$. \\ Nico Dragano ${ }^{4}$ \\ ${ }^{1}$ Fachgebiet 28, Soziale Determinanten der Gesundheit, Robert Koch-Institut, Berlin, Deutschland \\ ${ }^{2}$ Universität Bielefeld, Bielefeld, Deutschland \\ ${ }^{3}$ Martin-Luther-Universität Halle-Wittenberg, Halle-Wittenberg, Deutschland \\ ${ }^{4}$ Heinrich-Heine-Universität Düsseldorf, Düsseldorf, Deutschland \\ ${ }^{5}$ Medizinischen Fakultät Mannheim der Ruprecht-Karls-Universität Heidelberg, Heidelberg, Deutschland
}

\section{Soziale Ungleichheit und Gesundheit}

\section{Stand und Perspektiven der sozialepidemio- logischen Forschung in Deutschland}

In den letzten 30 Jahren haben zahlreiche empirische Studien gezeigt, dass auch in Deutschland, trotz des hohen allgemeinen Wohlstandes und der umfassenden sozialen Sicherungssysteme, ein enger Zusammenhang zwischen dem sozialen Status und der Gesundheit besteht. Dieser Zusammenhang lässt sich gerade in Bezug auf schwerwiegende chronische Erkrankungen und Beschwerden, die oftmals mit weitreichenden Beeinträchtigungen der Alltagsbewältigung und der gesundheitsbezogenen Lebensqualität einhergehen, sowie mit Blick auf die vorzeitige Sterblichkeit beobachten (siehe empirische Übersichten bei [1-4]). Die sozialen Unterschiede in der Morbidität und Mortalität werden auch auf den Begriff der „gesundheitlichen Ungleichheit" (engl.: "health inequality") gebracht und als eine extreme Ausprägungsform sozialer Ungleichheit verstanden, die in einem auf Solidarität und Chancengerechtigkeit aufbauenden Wohlfahrtsstaat eine große gesellschaftspolitische Herausforderung darstellt [5].

In den letzten Jahren hat sich innerhalb der Public-Health- und der epidemiologischen Forschung mit der Sozialepidemiologie eine eigenständige Forschungsrichtung etabliert, die sich nach eigenem Verständnis mit der sozialen Verteilung von Gesundheitschancen und Krankheitsrisiken sowie den sozialen Determinanten der Gesundheit befasst [6,
7]. Neben Impulsen aus den Vereinigten Staaten, Großbritannien, Skandinavien und einzelnen anderen europäischen Ländern, in denen die Sozialepidemiologie schon länger etabliert ist, wurde die Entwicklung in Deutschland maßgeblich durch Aktivitäten internationaler Organisationen, insbesondere der Weltgesundheitsorganisation (WHO), beeinflusst. $\mathrm{Zu}$ verweisen ist unter anderem auf die WHO-Rahmenprogramme Health for All und Health 2020, in denen die Verringerung der gesundheitlichen Ungleichheit zwischen und innerhalb der Länder als ein übergeordnetes Gesundheitsziel beschrieben wird [8], sowie die von der WHO eingerichtete Commission on Social Determinants of Health, die in ihrem unter der Leitung von Sir Michael Marmot erarbeiteten Abschlussbericht die sozialen Determinanten der Gesundheit als die zentralen Ansatzpunkte der politischen Bemühungen um eine Verbesserung der Bevölkerungsgesundheit und einen Abbau der gesundheitlichen Ungleichheiten herausstellt [9].

Nachfolgend werden Stand und Perspektiven der sozialepidemiologischen Forschung in Deutschland beschrieben. Zunächst wird auf die Entwicklung und die aktuelle Situation der Sozialepidemiologie eingegangen und dabei auch ein Bezug zum internationalen Kontext hergestellt. Im Anschluss wird auf den empirischen Forschungsstand eingegangen, der sich seit Mitte der 1980er-Jahre ständig weiterentwickelt hat. Außerdem werden mehrere Modelle vorgestellt, die zur Systematisierung des empirischen Forschungsstandes beitragen und Anknüpfungspunkte für die Erklärung der gesundheitlichen Ungleichheit aufzeigen. Davon ausgehend werden abschließend einige wichtige Herausforderungen und Perspektiven für die sozialepidemiologische Forschung diskutiert: die Berücksichtigung der Lebenslaufperspektive, die Analyse epigenetischer Mechanismen, die Einbeziehung von Menschen mit Migrationshintergrund, die Verbindung zur Versorgungsforschung, die Untersuchung regionaler und sozialräumlicher Ungleichheiten sowie die Ausweitung der Analysen auf den internationalen Vergleich.

\section{Etablierung der Sozialepidemiologie als eigenständige Forschungsrichtung}

Die Diskussion über soziale Unterschiede in der Gesundheit und Lebenserwartung hat in Deutschland eine lange Tradition. Im Grunde reicht diese bis zu Rudolf Virchow und Salomon Neumann zurück, die bereits Mitte des 19. Jahrhunderts auf die gesellschaftlichen Ursachen von Krankheit und vorzeitiger Sterblichkeit hingewiesen und staatliche 
Maßnahmen zum Schutz der Gesundheit gefordert haben [10]. Mit Beginn des Ersten Weltkriegs kam die an den sozialen Problemen ansetzende Forschungsrichtung der Medizin allerdings zum Erliegen und wurde auch nach dem Ende des Zweiten Weltkriegs zunächst nicht wieder aufgegriffen. Die Wiederbelebung des Themas setzte erst mit der Veröffentlichung des Black Reports in Großbritannien im Jahr 1980 ein [11]. Dieser Bericht wurde von einer Arbeitsgruppe um Sir Douglas Black, den Präsidenten des „Royal College of Physicians“, erstellt und zeigte gravierende soziale Morbiditäts- und Mortalitätsunterschiede in der englischen und walisischen Bevölkerung auf. Der Black Report und der einige Jahre später unter dem Titel "The Health Divide“ [12] veröffentlichte Nachfolgebericht erzielten eine große öffentliche Resonanz und lösten zunächst in Großbritannien, dann auch in vielen anderen Ländern eine rege Forschungstätigkeit aus.

In Deutschland setzte diese Entwicklung verspätet ein. Mit Beginn der 1990er-Jahre hat das Forschungsinteresse an der sozial ungleichen Verteilung des Erkrankungs- und Sterberisikos dann aber kontinuierlich zugenommen. Ein Beleg hierfür ist, dass gesundheitswissenschaftlich orientierte Fachzeitschriften wiederholt Schwerpunkthefte zur gesundheitlichen Ungleichheit herausgegeben haben. Neben dem vorliegenden Schwerpunktheft des Bundesgesundheitsblatts ist auf Hefte der Zeitschriften „Sozial- und Präventivmedizin (heute: International Journal of Public Health)“ (1993, Jg. 38, Heft 3) und der „Zeitschrift für Gesundheitswissenschaften (heute: Journal of Public Health)“ (2000, Jg. 6, Heft 3) als frühere Beispiele zu verweisen. Im Jahr 2007 hat auch das Deutsche Ärzteblatt ein Schwerpunktheft mit dem Titel "Armut und Gesundheit" veröffentlicht, das Teil einer gemeinsamen Aktion von 230 internationalen medizinischen Fachzeitschriften war („Global Theme Issue on Poverty and Human Development", 2007, Jg. 104, Heft 42). Zudem sind mehrere Sammelbände und Monografien erschienen, die einen Überblick über den Stand der Theoriebildung und der empirischen Forschung ver- mitteln, wie z. B. „Krankheit und soziale Ungleichheit" [13], „Soziale Ungleichheit und Gesundheit" [1], ,Sozial-Epidemiologie “ [6], „Müssen Arme früher sterben?" [14], „Gesundheitliche Ungleichheit" [3] und "Health inequalities" [15]. Das zunehmende Interesse spiegelt sich ebenfalls in einer Reihe von Fachtagungen und Konferenzen zur gesundheitlichen Ungleichheit wider. Zu nennen sind hier insbesondere die seit 1995 jährlich stattfindenden Kongresse „Armut und Gesundheit" in Berlin, die zwischen 2005 und 2009 ausgerichteten Kongresse "Health Inequalities" in Bielefeld und Berlin sowie die an wechselnden Orten durchgeführten Jahrestagungen der Deutschen Gesellschaft für Medizinische Soziologie (DGMS), der Deutschen Gesellschaft für Sozialmedizin und Prävention (DGSMP) und der Deutschen Gesellschaft für Epidemiologie (DGEpi), die sich regelmäßig mit der gesundheitlichen Ungleichheit und den sich daraus ergebenden gesellschaftlichen Herausforderungen befassen. Eine wichtige Rolle bei der Koordination der verschiedenen Veranstaltungen spielt die im Jahr 1997 gegründete AG Sozialepidemiologie, die Wissenschaftlern aus verschiedenen Fachgesellschaften eine gemeinsame Arbeitsplattform bietet. $\mathrm{Zu}$ verweisen ist außerdem auf die Sektion "Medizin- und Gesundheitssoziologie" innerhalb der Deutschen Gesellschaft für Soziologie (DGS), auch weil diese die Verbindung zur Soziologie herstellt. Die Institutionalisierung der Sozialepidemiologie an den deutschen Hochschulen erfolgt vor allem über die Gesundheitswissenschaften und die Humanmedizin, vereinzelt auch über die Sozialwissenschaften. Darüber hinaus gingen in den letzten Jahren wichtige Impulse von einigen außeruniversitären Forschungseinrichtungen aus, z. B. dem Robert Koch-Institut in Berlin, dem Helmholtz Zentrum in München und dem LeibnizInstitut für Präventionsforschung und Epidemiologie in Bremen. Dass das Thema gesundheitliche Ungleichheit immer wieder in den Blickpunkt der Öffentlichkeit gerät und mittlerweile seitens der Politik eine stärkere Resonanz erfährt, ist auch der Gesundheitsberichterstattung auf Bundes- und Länderebene zu verdanken. Im Rahmen der Gesundheitsberichterstattung des Bundes werden mittlerweile regelmäßig Berichte veröffentlicht, die sich mit der sozial ungleichen Verteilung der Gesundheitschancen und Erkrankungsrisiken befassen [16-19]. Inzwischen wird das Thema auch in anderen Berichtswesen behandelt, wie z. B. der Armuts- und Reichtumsberichterstattung der Bundesregierung [20, 21] und den Gutachten des Sachverständigenrates zur Begutachtung der Entwicklung im Gesundheitswesen [22, 23].

\section{Empirischer Forschungsstand in Deutschland}

Erste umfassende empirische Analysen der sozialen Ungleichheit im Krankheitsgeschehen wurden in Deutschland Mitte der 1980er-Jahre im Rahmen des MONICA-Projektes (Monitoring Trends and Determinants in Cardiovascular Disease) und der Deutschen Herz-Kreislauf-Präventionsstudie (DHP) durchgeführt. Beide Studien hatten einen Schwerpunkt auf kardiovaskulären Erkrankungen und Risikofaktoren. Die im Anschluss an die DHP-Studie vom Robert Koch-Institut durchgeführten Gesundheitssurveys erweiterten das Themenspektrum auf die subjektive Gesundheit, die gesundheitsbezogene Lebensqualität, das Gesundheitsverhalten jenseits der klassischen verhaltenskorrelierten Risikofaktoren und die Gesundheitsversorgung. Aktuell stellen die vom Robert Koch-Institut im Rahmen des Gesundheitsmonitorings durchgeführten Surveys "Gesundheit in Deutschland aktuell" (GEDA), „Studie zur Gesundheit Erwachsener in Deutschland" (DEGS) und „Studie zur Gesundheit von Kindern und Jugendlichen in Deutschland" (KiGGS) eine wichtige Datengrundlage für die sozialepidemiologische Forschung bereit. Daneben können weitere Gesundheitssurveys, die häufig thematisch oder in Bezug auf die Altersgruppen stärker eingegrenzt sind, genutzt werden, z. B. der Epidemiologische Suchtsurvey (ESA) des Instituts für Therapieforschung (IFT), die Drogenaffinitätsstudie (DAS) der Bundeszentrale für gesundheitliche Aufklärung (BZgA) und die Studie Health-Behaviour in School-aged Children (HBSC) der 
Weltgesundheitsorganisation (WHO). Zusätzliche Analysemöglichkeiten eröffnen sich über epidemiologische Studien, wie z. B. die Heinz Nixdorf Recall-Studie, die Study of Health in Pomerania (SHIP), die inzwischen in der Feldphase befindliche Nationale Kohorte (NAKO) sowie die 2013 gestartete sozialepidemiologische Geburtskohortenstudie Gesundheit von Babys und Kindern in Bielefeld (BaBi). Darüber hinaus kommt sozialwissenschaftlichen Erhebungen, z. B. dem Sozio-oekonomischen Panel (SOEP) und der Allgemeinen Bevölkerungsbefragung der Sozialwissenschaften (ALLBUS), sowie amtlichen Statistiken, z. B. dem Mikrozensus und der Statistik Leben in Europa (EU SILC), ein hoher Stellenwert zu. In diesen Erhebungen stehen zwar weniger Gesundheitsinformationen zur Verfügung, dafür werden die Lebensbedingungen sehr differenziert erfasst. Zunehmend genutzt werden auch die Daten der Sozialversicherungsträger, vor allem der gesetzlichen Krankenversicherung (GKV) und der Deutschen Rentenversicherung Bund, da die Zugangswege zu diesen Daten, u. a. durch die stärkere Förderung von Kooperationen mit Forschungseinrichtungen und die Einrichtung von Forschungsdatenzentren, in den letzten Jahren erheblich verbessert wurden.

Die vorliegenden Forschungsergebnisse deuten in großer Übereinstimmung darauf hin, dass Personen mit niedrigem sozialen Status häufiger von Krankheiten und Beschwerden betroffen sind als Personen mit höherem sozialen Status ([1-3, 16]; - Abb. 1). Die Unterschiede zwischen den Statusgruppen fallen gerade in Bezug auf schwerwiegende chronische Erkrankungen wie Herzinfarkt, Schlaganfall, Diabetes mellitus oder chronischobstruktive Lungenerkrankung deutlich aus. Für Herzinfarkt und Schlaganfall kann z. B. von einem 2-bis 3-fach erhöhten Erkrankungsrisiko in der niedrigen im Verhältnis zur hohen Statusgruppe gesprochen werden [24-26]. Werden anstelle von Survey-Daten die auf ärztlichen Diagnosen basierenden Abrechnungsdaten der gesetzlichen Krankenversicherung herangezogen, dann fallen die sozialen Unterschiede bei Herz-Kreislauf-Erkrankungen noch stärker aus [27,

Bundesgesundheitsbl 2016 · 59:153-165 DOI 10.1007/s00103-015-2275-6

(c) Springer-Verlag Berlin Heidelberg 2015

T. Lampert · M. Richter · S. Schneider · J. Spallek · N. Dragano

\section{Soziale Ungleichheit und Gesundheit. Stand und Perspektiven der sozialepidemiologischen Forschung in Deutschland}

\section{Zusammenfassung}

Soziale Unterschiede in der Morbidität und Mortalität stellen seit jeher einen zentralen Gegenstand der Public-HealthForschung dar. In den letzten Jahren ist ein zunehmendes Forschungsinteresse festzustellen, das in der breiten Öffentlichkeit und auch in der Politik einen deutlichen Widerhall findet. Der vorliegende Beitrag beschreibt die Entwicklung und Etablierung der Sozialepidemiologie in Deutschland und stellt den aktuellen empirischen Forschungsstand dar. Außerdem werden verschiedene Modelle zur Erklärung der gesundheitlichen Ungleichheit beschrieben. Auf dieser Grundlage werden anschließend ausgewählte Herausforderungen und Perspektiven der sozialepidemiologischen Forschung aufgezeigt und anhand dieser verdeutlicht, weshalb die Analyse der sozialen Unterschiede in der Morbidität und Mortalität auch künftig eine zentrale Aufgabe der Public-Health-Forschung im nationalen sowie internationalen Kontext darstellen wird.

Schlüsselwörter

Sozialepidemiologie · Sozialstatus .

Soziale Ungleichheit •

Gesundheitliche Ungleichheit · Public Health

\section{Social inequality and health. Status and prospects of socio-epidemiological research in Germany}

\section{Abstract}

Social differences in morbidity and mortality have always been a central topic in public health research. In recent years, there has been a growing research interest that has clearly resonated with the general public and the political arena as well. This article describes the development and establishment of social epidemiology in Germany and presents the current status of research. In addition, it describes different models for explaining health inequalities. On this basis, selected

28]. Außerdem konnten auf Grundlage der GKV-Daten soziale Unterschiede bei Krebserkrankungen, wie z. B. Magen-, Darm- und Lungenkrebs, gezeigt werden [29]. Auch psychische Erkrankungen und Störungen treten in der niedrigen Statusgruppe vermehrt auf. Dies gilt z. B. für Depressionen, Angststörungen und Substanzstörungen [30, 31]. Mit Blick auf die im Krankheitsspektrum vorherrschenden Erkrankungen sind nur wenige Ausnahmen auszumachen. Dazu zählen allergische Erkrankungen, die häufiger in den höheren Statusgruppen anzutreffen sind [32, 33]. Auch für Brustkrebs wird bisweilen ein verstärktes Vorkommen in den höheren Statusgruppen berichtet, die Ergebnisse hierzu sind aber nicht einheitlich $[28,29]$. challenges and prospects of socio-epidemiological research are demonstrated. The reason why the analysis of social differences in morbidity and mortality will continue to be a key task of public health research in the national and international context in the future is also explained.

\section{Keywords}

Social epidemiology · Social status . Social inequality $\cdot$ Health inequality . Public health
Deutliche Unterschiede zuungunsten der niedrigen Statusgruppen zeichnen sich darüber hinaus im Auftreten von Unfallverletzungen, Behinderungen und funktionellen Einschränkungen ab [16, 34]. Entsprechend unterschiedlich fällt die Selbsteinschätzung des allgemeinen Gesundheitszustands und der gesundheitsbezogenen Lebensqualität aus. Zum Teil kommen die Statusunterschiede in der subjektiven Gesundheit sogar noch stärker zum Ausdruck, was als Hinweis auf Unterschiede in Bezug auf gesundheitsbezogene Einstellungen, Wahrnehmungen und Überzeugungen sowie personale und soziale Bewältigungsressourcen verstanden wird [35, 36].

Viele der Erkrankungen und Beschwerden, in deren Verbreitung sich soziale Unterschiede abzeichnen, können 


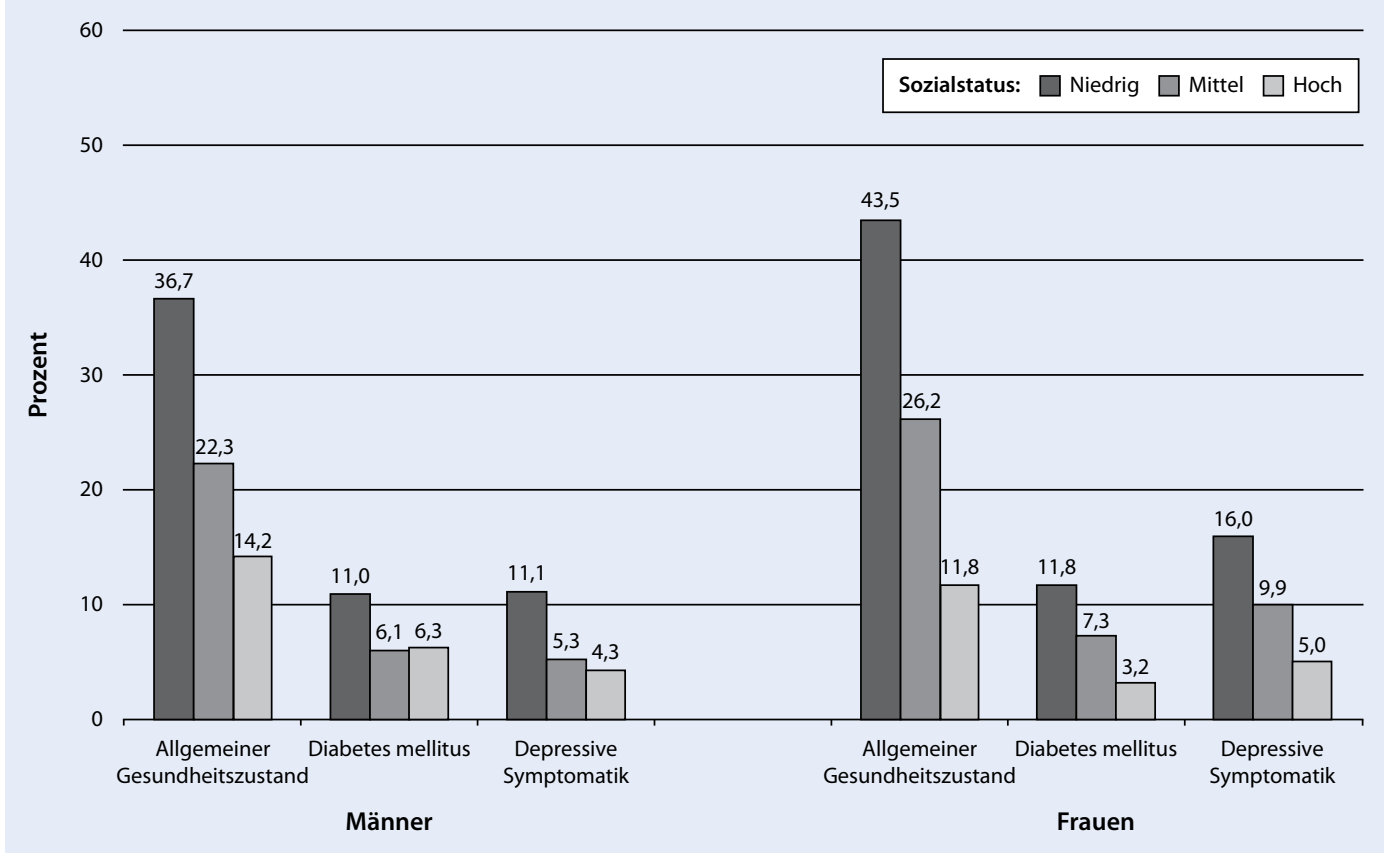

Abb. $1 \varangle$ Allgemeiner Gesundheitszustand („,mittelmäßig", ,,schlecht" oder "sehr schlecht"), Diabetes mellitus (Lebenszeitprävalenz) und Depressive Symptomatik (Patient Health Questionnaire, PHQ-9>10) nach sozialem Status in der 18- bis 79-jährigen Bevölkerung. Datenbasis: Studie zur Gesundheit Erwachsener in Deutschland (DEGS1), 2008-2011 [28] auf Risikofaktoren zurückgeführt werden, die im Zusammenhang mit dem Gesundheitsverhalten zu sehen sind. Große Bedeutung kommt dabei dem Rauchen $\mathrm{zu}$, das in den niedrigen Statusgruppen deutlich stärker verbreitet ist. Zwar lässt sich in den letzten Jahren in allen Statusgruppen ein Rückgang des Rauchens beobachten, die sozialen Unterschiede sind aber weitgehend erhalten geblieben [37]. Bezüglich des Alkoholkonsums ergibt sich kein eindeutiges Bild. Gerade bei Frauen zeigt sich, dass auch in den höheren Statusgruppen ähnlich häufig oder sogar häufiger regelmäßig und auch riskant Alkohol konsumiert wird. In Bezug auf die Alkoholabhängigkeit und alkoholbedingte Erkrankungen sind aber zumindest bei Männern deutliche Unterschiede zuungunsten der niedrigen Statusgruppen festzustellen [38]. Auch mit Blick auf das Bewegungsverhalten ist eine differenzierte Betrachtungsweise angezeigt. Die höheren Statusgruppen treiben häufiger Sport, sind aber in ihrem Alltag seltener körperlich aktiv, da sie häufiger sitzenden Tätigkeiten nachgehen [39]. Zu berücksichtigen ist dabei, dass die höhere Alltagsaktivität in den niedrigen Statusgruppen der Gesundheit nicht immer zuträglich ist, da sie oftmals mit körperlicher Überbeanspruchung und Fehlbelastung sowie folglich mit Risiken für die Gesundheit ein- hergeht [40, 41]. Darüber hinaus lässt sich feststellen, dass sich die Angehörigen der niedrigen Statusgruppen ungesünder ernähren. Festmachen lässt sich dies unter anderem an einer insgesamt höheren Kalorienzufuhr, einer oftmals nicht ausgewogenen Ernährungsweise und einem häufigeren Verzehr von fett- und zuckerhaltigen Lebensmitteln [42]. Auch in der Inanspruchnahme des Gesundheitswesens treten deutliche soziale Unterschiede zutage. Dies gilt insbesondere für präventive und gesundheitsfördernde Angebote wie die Krebsfrüherkennungsuntersuchungen, den GesundheitsCheck-up und die Teilnahme an kassenfinanzierten Angeboten zur Gesundheitsförderung [43-45].

Die sozialen Unterschiede in Bezug auf Erkrankungen, Beschwerden und die zugrunde liegenden Risikofaktoren kumulieren letztlich in einem erhöhten vorzeitigen Sterberisiko in den sozial benachteiligten Bevölkerungsgruppen. Mit Blick auf das Einkommen sprechen die vorliegenden Studien für eine um fünf bis über zehn Jahre verringerte mittlere Lebenserwartung bei Geburt bei Personen mit niedrigen im Vergleich zu Personen mit hohen Einkommen [46, 47]. Betrachtet man nur die Lebensjahre, die bei guter Gesundheit verbracht werden, die sogenannte gesunde Lebenserwartung, dann fallen die Unterschiede noch größer aus (• Tab. 1).

Die sozialen Unterschiede in der Gesundheit treten nicht erst im Erwachsenenalter hervor, sondern zeichnen sich bereits bei Kindern und Jugendlichen deutlich ab. Die KiGGS- und HBSCStudie, aber auch die Einschulungsuntersuchungen des Öffentlichen Gesundheitsdienstes und andere Erhebungen machen deutlich, dass der soziale Status der Familie beinahe alle Gesundheitsund Entwicklungsbereiche im Kindesund Jugendalter beeinflusst. Kinder und Jugendliche aus Familien mit niedrigem Sozialstatus haben z. B. ein deutlich erhöhtes Risiko für Verhaltensauffälligkeiten, einschließlich ADHS und Essstörungen, für Bewegungsmangel und Adipositas, sie ernähren sich ungesünder und schätzen ihre eigene gesundheitsbezogene Lebensqualität schlechter ein $[34,48]$. Bereits rund um die Geburt zeichnen sich deutliche soziale Unterschiede $a b$, was sich unter anderem daran festmachen lässt, dass Kinder aus sozial benachteiligten Familien häufiger in der Schwangerschaft dem mütterlichen Rauchen ausgesetzt sind, zu einem geringeren Anteil gestillt werden und seltener an den U-Untersuchungen teilnehmen [49, 50]. Diese Unterschiede können aus einer Lebenslaufperspektive als frühe Weichenstellungen begriffen 
Tab. 1 Lebenserwartung bei Geburt und gesunde Lebenserwartung bei Geburt nach Einkommen (in Jahren). Datenbasis: Sozio-oekonomisches Panel und Periodensterbetafeln 1995-2005 [46]

\begin{tabular}{|c|c|c|c|c|}
\hline & \multicolumn{2}{|c|}{ Lebenserwartung bei Geburt } & \multicolumn{2}{|c|}{ Gesunde Lebenserwartung ${ }^{a}$ bei Geburt } \\
\hline & Männer & Frauen & Männer & Frauen \\
\hline \multicolumn{5}{|c|}{ Netto-Äquivalenzeinkommen ${ }^{\mathbf{b}}$} \\
\hline$<60 \%$ & 70,1 & 76,9 & 56,8 & 60,8 \\
\hline $60 \%-<80 \%$ & 73,4 & 81,9 & 61,2 & 66,2 \\
\hline $80 \%-<100 \%$ & 75,2 & 82,0 & 64,5 & 67,1 \\
\hline $100 \%-<150 \%$ & 77,2 & 84,4 & 66,8 & 69,1 \\
\hline$\geq 150 \%$ & 80,9 & 85,3 & 71,1 & 71,0 \\
\hline
\end{tabular}

werden, die sich noch im mittleren und höheren Lebensalter in erhöhten Erkrankungsrisiken, z. B. in Bezug auf HerzKreislauf- und Atemwegserkrankungen, widerspiegeln [49, 51].

Darüber hinaus machen die vorliegenden Forschungsergebnisse deutlich, dass die sozialen Unterschiede im Gesundheitszustand und in der Lebenserwartung in allen Altersgruppen nicht nur beim Vergleich der am schlechtesten mit den am besten gestellten Statusgruppen festzustellen sind. Vielmehr sind auch Unterschiede zwischen den niedrigen und mittleren Statusgruppen sowie zwischen den mittleren und höheren Statusgruppen $\mathrm{zu}$ beobachten. Zumindest in Bezug auf bestimmte Bereiche der Gesundheit kann davon gesprochen werden, dass die Erkrankungsrisiken umso geringer bzw. die Gesundheitschancen umso besser sind, je höher der soziale Status ist. Dieses Verteilungsmuster wird als sozialer Gradient oder Statussyndrom bezeichnet und damit auch in Bezug auf die Verteilung der Erkrankungsrisiken bzw. Gesundheitschancen die Bedeutung von relativen Ungleichheiten, die sich über das gesamte Spektrum der Statuslagen abzeichnen, betont $[52,53]$.

\section{Erklärung der gesundheitlichen Ungleichheit}

Neben der Beschreibung stellt die Erklärung der gesundheitlichen Ungleichheit einen Gegenstand der sozialepidemiologischen Forschung dar. Ein wichtiger Meilenstein war auch in dieser Hinsicht der Black Report, in dem die beobachteten sozialen Unterschiede in der Morbidität und Mortalität mittels struktureller, materieller, kultureller und verhaltensbezogener Determinanten erklärt werden. Als strukturelle Determinanten werden vor allem die körperlichen und psychischen Wirkungen von Arbeits- und Wohnbedingungen bezeichnet. Materielle Determinanten zielen auf ungleich verteilte materielle Ressourcen, insbesondere Einkommen und Vermögen, und resultierende ökonomische Deprivationslagen ab. Unter kulturellen Determinanten werden zum einen eher direkte Bildungseffekte wie etwa medizinisches Wissen und zum anderen eher indirekte Effekte kulturellen Kapitals wie etwa die durch Bildung erworbene Befähigung zur Selbstdisziplinierung bezüglich gesundheitsschädlicher Verhaltensweisen und die Handlungskompetenz bei persönlichen Belastungssituationen gefasst [54]. Die verhaltensbezogenen Determinanten schließlich beziehen sich auf statusspezifisch sozialisierte und durch die jeweiligen materiellen Lebensbedingungen geprägte gesundheitsrelevante Verhaltensweisen und Lebensstile.

Damit brachte der Black Report neben individuellem Verhalten auch sozial vermittelte Ursachen gesundheitlicher Ungleichheit in die Diskussion ein. In den Jahren darauf folgte dann durch verschiedene Autoren zunächst eine weitere Differenzierung, Strukturierung und Einordnung dieser Ursachen entlang der Mikro-, Meso- und Makroebene. Vor allem aber verlagerten sich die Hauptströmungen der Diskussion weg von einer individualistischen Risikofaktorenmedizin, zunächst hin in Richtung Meso- und später weiter in Richtung Makroebene. Ein Beispiel hier- für ist das im deutschen Sprachraum weit verbreitete Modell zur Erklärung gesundheitlicher Ungleichheit von Mielck und Elkeles [1, 55]. Dieses Modell konstatiert zum einen eine sozial ungleiche Verteilung an Wissen, Macht, Geld und Prestige und zum anderen sozial bedingte Unterschiede im gesundheitsrelevanten Verhalten. Auf der Mesoebene werden sozial bedingte Unterschiede erstens in der Arbeitsund Wohnsituation (vor allem Arbeitsstress und Wohnumgebung), zweitens in den Bewältigungsressourcen (soziale Unterstützung etwa am Arbeitsplatz und kompensatorische Erholungsmöglichkeiten etwa in der Wohnumgebung) sowie drittens in der medizinischen Versorgung identifiziert.

Darüber hinaus berücksichtigt das Modell die bereits im Black Report diskutierte Möglichkeit, dass gesundheitsbedingte Beeinträchtigungen einen sozialen Aufstieg erschweren respektive einen sozialen Abstieg begünstigen können. Allerdings wird mit Blick auf die vorliegenden Studien die Bedeutung eines solchen Selektionseffektes für die Erklärung der gesundheitlichen Ungleichheit als eher gering eingeschätzt $[55,56]$.

In den folgenden Jahren entwickelten sich Theorie und Empirie hinsichtlich dieser drei Richtungen weiter: Erstens wurden psychosoziale Faktoren, wie Stress und Unterstützung am Arbeitsplatz, mittels unterschiedlicher Modelle untersucht. Insbesondere Siegrists Modell beruflicher Gratifikationskrisen erwies sich dabei für die Erklärung gesundheitlicher Ungleichheit als anschlussfähig [57]. Zweitens wurde auch die Bedeutung der natürlichen und gebauten Umwelt für die Entstehung gesundheitlicher Un- 


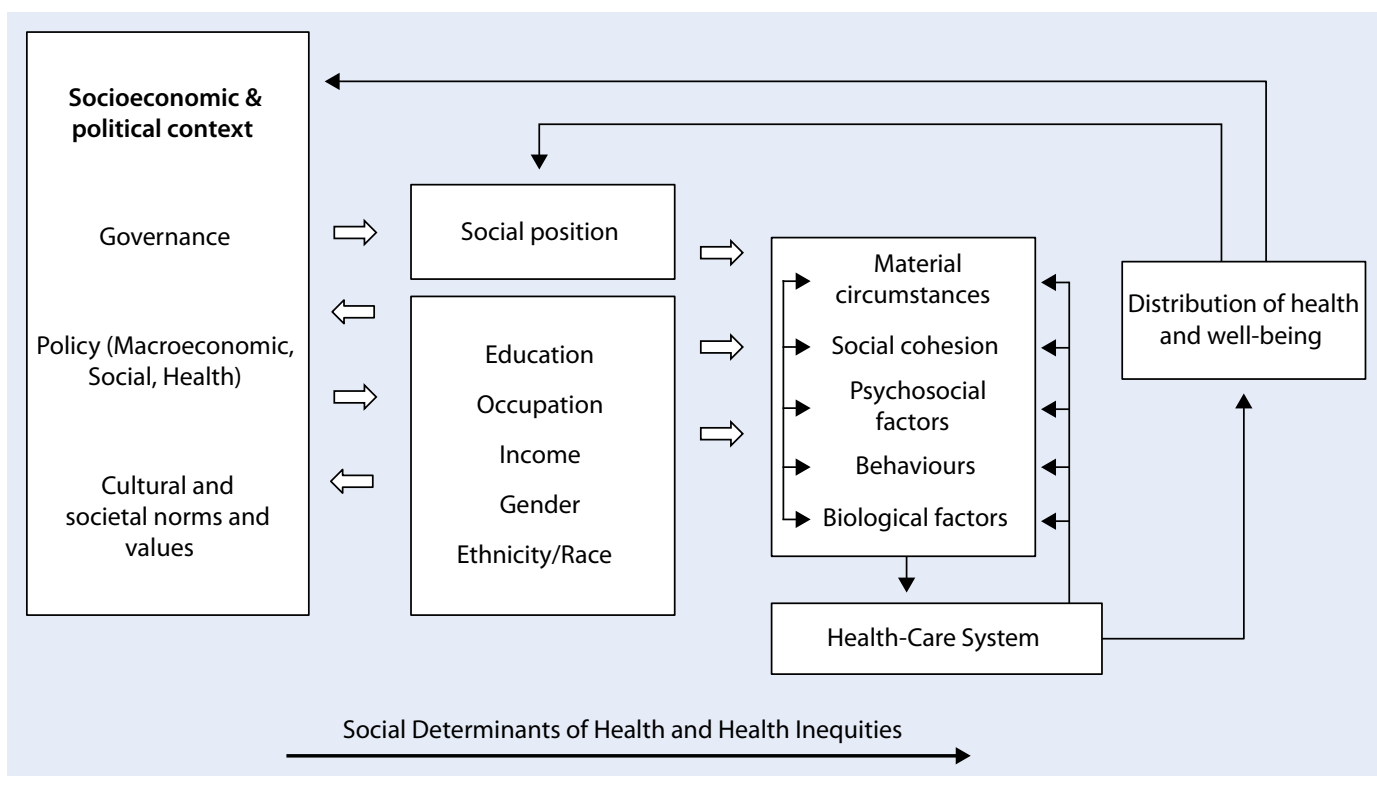

Abb. $2<$ Framework der WHO-Kommission „Social Determinants of Health" zur Erklärung der gesundheitlichen Ungleichheit $[9,60]$ gleichheiten zunehmend erkannt und beforscht [58]. Drittens entstand ebenso ein eigener Forschungszweig zur Frage, inwiefern Angebot, Versorgung und Inanspruchnahme medizinischer Leistungen Ungleichheiten mitbedingen $[59,60]$.

In den letzten Dekaden war der Theoriediskurs also geprägt von einer Verortung der Ursachen gesundheitlicher Ungleichheit von „proximal nach distal“ und von einer zunehmenden Differenzierung der Einflussgrößen vorrangig auf der Mesoebene. Dabei erfolgte auch eine Ausweitung der Betrachtung auf „horizontale“ Ungleichheiten, etwa hinsichtlich des Geschlechts, der Nationalität und des Migrationshintergrunds, sowie auf die Entwicklung im Lebenslauf [61].

Aktuelle Erklärungsansätze gesundheitlicher Ungleichheit stellen nunmehr makrosoziologische Einflüsse in den Vordergrund. Das wohl bekannteste Modell ist derzeit die CSDH-Framework der Commission on Social Determinants of Health der WHO (• Abb. 2). Die WHO kommt zu dem Ergebnis, dass viele derzeitige Gesundheitssysteme gesundheitliche Disparitäten zwischen privilegierten und benachteiligten gesellschaftlichen Gruppen eher vergrößern als verringern. Die WHO identifiziert dabei auf der Makroebene - neben Globalisierungsprozessen - die makroökonomische Politik, die Sozialpolitik, das öffentliche Bildungs-, Gesundheits- und Sozialversicherungssystem ebenso wie die politischen Entscheider und Regierenden sowie das gesellschaftliche Wertesystem als strukturelle Determinanten gesundheitlicher Ungleichheit. Vice versa könnten nationale Gesundheitssysteme per se zu einer Verringerung gesundheitlicher Ungleichheiten beitragen, indem sie die intersektorale Zusammenarbeit intensivieren und dabei soziale Beteiligung und soziales Empowerment auf Bürger- und Institutionenebene nutzen, um die alltäglichen Lebensbedingungen $\mathrm{zu}$ verbessern und einer ungerechten Verteilung von Ressourcen entgegenzuwirken $[9,62]$.

In einem von Pons-Vigués und Kollegen jüngst veröffentlichten Modell zur Erklärung gesundheitlicher Ungleichheiten auf kommunaler Ebene richten die Autoren ihr Hauptaugenmerk ebenfalls auf makrosystemische Einflüsse und betonen die Rolle von Politik und Regierung sowie nationaler und globaler Entwicklungen auf die gesundheitliche Ungleichheit [63].

\section{Forschungsperspektiven}

Die Forschung zur sozialen Ungleichheit von Gesundheit und Krankheit beschäftigt sich mit einer komplexen Gemengelage sozialer, ökonomischer, psychosozialer und biologischer Prozesse. Auch wenn mittlerweile viel über die Zusammenhänge und die zugrunde liegenden Prozesse und Mechanismen, die zur Produktion und Reproduktion der gesundheitlichen Ungleichheit beitragen, bekannt ist, so bleibt doch ein weites Feld an offenen Fragen. Im Folgenden werden deshalb einige ausgewählte Forschungsperspektiven beschrieben, die für die Weiterentwicklung und künftige Ausrichtung der Sozialepidemiologie von großer Bedeutung sein dürften.

\section{Lebenslaufbezogene Analyse der gesundheitlichen Ungleichheit}

Die epidemiologische Lebenslaufforschung betrachtet Gesundheit als Ergebnis langfristiger biologischer, psychischer und sozialer Prozesse [64]. Ausgangspunkt für die Entwicklung einer lebenszeitlichen Perspektive waren Analysen, die Zusammenhänge zwischen frühkindlichen Risikofaktoren, z. B. einem geringen Geburtsgewicht, und einer frühzeitigen Mortalität im höheren Lebensalter fanden [65]. Mittlerweile sind zahlreiche Zusammenhänge bekannt, bei denen sowohl biologische als auch psychosoziale Risikofaktoren, die in jungen Jahren auftreten, das Erkrankungsgeschehen im späteren Leben in vielfältiger Weise beeinflussen [66].

Die Erklärung dieser Zusammenhänge ist aufgrund der zeitlichen Abhängigkeiten und der Vielzahl beteiligter Faktoren äußerst komplex. Daher sollen hier nur zwei zentrale Annahmen skizziert werden. Die erste An- 
nahme ist, dass "kritische Perioden“ existieren, in denen Weichenstellungen für die weitere gesundheitliche Entwicklung erfolgen. Diese Perioden sind bestimmte Phasen der physiologischen und psychischen Entwicklung, in denen eine erhöhte Vulnerabilität gegenüber Störungen der natürlichen Entwicklung besteht. Ein besonderer Stellenwert wird dabei der Schwangerschaft beigemessen, aber auch im Säuglings-, Kindes- und Jugendalter gibt es spezifische Phasen, in denen wichtige Entwicklungsaufgaben abgeschlossen sein sollten (z. B. Sprachentwicklung, Körperwachstum). Kommt es in diesen Phasen zu Störungen, dann können sich diese langfristig auswirken und noch im höheren Lebensalter zu einem erhöhten Risiko für Krankheiten und Funktionseinschränkungen führen [66].

Eine weitere Annahme ist, dass es weniger einmalige Ereignisse, sondern kumulative und zeitlich andauernde Prozesse sind, die die Gesundheit im Lebenslauf prägen [67]. Neben der Dauer kommt dabei auch der Intensität und dem Beginn der Risikoexposition ein hoher Stellenwert zu. Für viele Risikofaktoren, wie z. B. Rauchen, Mangel- und Fehlernährung, Übergewicht und Adipositas sowie geringe körperlich-sportliche Aktivität, konnte inzwischen gezeigt werden, dass die Auswirkungen auf die Gesundheit umso stärker sind, je früher im Leben sie auftreten, je stärker sie ausgeprägt sind und je länger sie wirken [68, 69].

Der Zusammenhang zwischen sozialer Position und Gesundheit im Lebenslauf begründet sich dadurch, dass zahlreiche gesundheitliche Belastungen in frühen Lebensphasen sozial ungleich verteilt sind. So lassen sich bei Faktoren wie dem kindlichen Übergewicht oder Entwicklungsstörungen im Kleinkindalter deutliche Unterschiede zwischen den sozialen Gruppen erkennen, wobei eben Kinder aus ärmeren und schlechter gebildeten Familien häufiger betroffen sind [17]. Diese sozial ungleiche Verteilung von Risikofaktoren schreibt sich bis in das Jugendalter fort, sodass die betroffenen Personen bereits beim Eintritt in das Berufsleben erhebliche gesundheitliche Beeinträchtigungen haben können.
Weitere Bedeutung bekommen diese Prozesse durch ihre Interaktion mit der sozialen Mobilität. Kinder aus benachteiligten Familien haben nicht nur ein höheres Risiko, gesundheitlichen Belastungen ausgesetzt zu sein, sondern sind zugleich auch im Hinblick auf ihre Bildungskarrieren benachteiligt. Ursächlich sind hier strukturelle Eigenheiten des Bildungssystems, die zur Folge haben, dass der Bildungserfolg in starkem Maße von der sozioökonomischen Position der Herkunftsfamilie abhängt [70]. Erreichen diese Jugendlichen ihrerseits niedrigere Bildungsabschlüsse, so ist die Wahrscheinlichkeit hoch, dass sie auch am Arbeitsmarkt eher niedrigere berufliche Positionen einnehmen werden. Über die Assoziation einer niedrigen beruflichen Position mit verschiedenen negativen gesundheitsrelevanten Einflüssen, wie z. B. einer prekären Beschäftigung, niedrigem Einkommen und höheren Arbeitsbelastungen, schreiben sich somit soziale und gesundheitliche Benachteiligungen fort [71].

Die vorliegenden empirischen Studien zur gesundheitlichen Ungleichheit im Lebenslauf stammen überwiegend aus dem amerikanischen, britischen und skandinavischen Raum. Es wäre wünschenswert, wenn auch die hierzulande zunehmend bessere Datenlage vermehrt für längsschnittliche, lebenslaufbezogene Analysen genutzt würde. Solche Analysen haben nicht nur einen Wert für die Grundlagenforschung, sondern machen zugleich deutlich, auf welche spezifischen Risikokonstellationen in einzelnen Lebensphasen sich eine zielgerichtete Prävention konzentrieren sollte.

\section{Bedeutung epigenetischer Mechanismen für die gesundheitliche Ungleichheit}

Zusammenhänge im Lebenslauf sind mittlerweile gut belegt, jedoch sind die zugrunde liegenden biologischen Mechanismen der Genese gesundheitlicher Ungleichheiten noch zu einem großen Teil unverstanden [72-75]. Das Wissen über die Produktion und Reproduktion gesundheitlicher Ungleichheiten im Lebenslauf hört damit - im übertragenen
Sinne - häufig an der Hautoberfläche auf. Im Gegenzug haben die molekulare Biologie und die Biomedizin distalen Einflussgrößen bislang sehr wenig Bedeutung geschenkt.

Erst in den letzten Jahren rückten naturwissenschaftliche Erkenntnisse und allen voran das Epigenom in den Fokus der Forschung über gesundheitliche Ungleichheiten [76-78]. Die Epigenetik als grundlagenwissenschaftliches Forschungsfeld innerhalb der Biologie untersucht Gen-Umwelt-Interaktionen. Dabei geht es im Kern um die Beobachtung, dass eine identische genetische Ausstattung zweier Lebewesen nicht zwangsläufig auch zur Ausprägung identischer Phänotypen führt. Sehr allgemein formuliert regelt das Epigenom, ob genetische Anlagen auch tatsächlich zum Tragen kommen (sog. Genexpression). Solche Einflüsse auf die Genexpression sind nicht statisch, sondern unterliegen vielfältigen Umwelteinflüssen, sodass in Abhängigkeit von den Lebensumständen biologische Prozesse in Gang gesetzt werden können - oder eben nicht.

Erste Studien über die Rolle sozialer Determinanten in der Genaktivität zeigen, dass Faktoren wie soziale Isolation, sozialer Stress und gerade der sozioökonomische Status tatsächlich mit der Genexpression assoziiert sind. So unterscheidet sich das Ausmaß der DNA-Methylierung, eines der zentralen epigentischen Mechanismen, deutlich nach dem Einkommen und der Bildung [79]. Ähnliche Assoziationen finden sich auch für andere (psycho)biologische Mechanismen, wie z. B. die Neurotransmission im Hirn oder die Zellalterung [72, 80, 81].

Die Verknüpfung sozialer Faktoren mit epigenetischen Prozessen kann dazu beitragen, Mehrebenenmodelle der Entstehung von Gesundheit und Krankheit $\mathrm{zu}$ entwickeln und zu testen und auf diese Weise die Kooperation zwischen Sozialund Naturwissenschaften zu stärken. Eine detaillierte Analyse des Zusammenspiels sozialer und biologischer Modelle erweitert nicht nur das Wissen über die Entstehung bestimmter Erkrankungen, sondern ist auch in der Lage, die bisherigen Erklärungsmodelle gesundheitlicher Ungleichheiten zu überprüfen und 
weiterzuentwickeln. Aktuell finden beispielsweise erste Übertragungen epigenetischer Erkenntnisse und Theorien in die lebenslaufbezogene Erforschung gesundheitlicher Ungleichheit statt [82, 83].

\section{Migration als Determinante gesundheitlicher Ungleichheit}

Migration wird mittlerweile als eine wichtige Determinante gesundheitlicher Ungleichheit in den westlichen Einwanderungsgesellschaften wahrgenommen, deren Einfluss aufgrund weiterer Zuwanderung vermutlich noch zunehmen wird. Die Sozialepidemiologie befasst sich daher seit einigen Jahren verstärkt mit dem Einfluss von Migration auf die Gesundheit der Bevölkerung. Einen wichtigen Zugang stellt dabei der Lebenslaufansatz dar, wobei der Lebenslauf von Migranten oftmals in drei Abschnitte unterteilt wird: die Phase vor, während und nach dem Migrationsereignis. In allen drei Phasen können spezifische Expositionen auf die Gesundheit der Migranten einwirken. Zu berücksichtigen ist allerdings, dass es zwischen Migranten je nach Herkunft, rechtlichem Status und Grund der Wanderung eklatante Unterschiede gibt [84].

Bereits in der Phase vor der Migration können Expositionen aufgetreten sein, die sich deutlich von denen der nichtmigrierten Bevölkerung unterscheiden. Besondere Bedeutung kommt dabei sicherlich Ereignissen zu, die letztlich den Ausschlag für die Migration gegeben haben, z. B. Naturkatastrophen, Armut oder bewaffnete Konflikte. Eine besonders kritische Phase, insbesondere bei Flüchtlingen, stellt der oft jahrelange Migrationsprozess dar, in dem diverse gesundheitliche Expositionen auftreten können. In der Phase nach der Migration werden dann kulturell geprägte Verhaltensunterschiede und die besondere soziale Lage der Migranten im Zielland bedeutsam. Vielfach wird argumentiert, dass gesundheitliche Nachteile von Migranten nach der Migration weitgehend eine Folge des niedrigen sozialen Status seien. In der Tat weisen Migranten überproportional häufig einen niedrigen Sozialstatus auf. Migranten haben häufiger eine schlechtere Schulbildung und berufliche Qualifikation als die Mehrheit der Bevölkerung im Zielland. Dies äußert sich dann beispielweise in einer höheren Arbeitslosenquote und im Durchschnitt schlechter bezahlten beruflichen Tätigkeiten. Hinzu kommen, zumindest bei Männern mit Migrationshintergrund, statusspezifische gesundheitsschädigende Verhaltensweisen, wie beispielsweise eine höhere Raucherquote [85]. Diese Belastungen und Risiken können mit schlechterer Gesundheit, besonders in zunehmendem Alter, assoziiert sein [86]. Nicht-migrierte Arbeitnehmer, die ähnliche Tätigkeiten ausführen, sind allerdings gesundheitlich ähnlich belastet. Es handelt sich hierbei also eher um ein statusspezifisches als um ein migrationsspezifisches Phänomen. Auch die Nachkommen von Migranten, die sogenannte zweite und dritte Generation, befinden sich oftmals noch in einer besonderen gesundheitlichen Situation, da auch hier die sozioökonomische Position der Eltern einen Einfluss hat. Das betrifft zum einen die materielle Lebenslage, in der Kinder aufwachsen, aber auch die kulturelle Prägung von Verhaltensweisen, wie z. B. der Esskultur oder der Umgang mit dem Gesundheitssystem. Aus sozialepidemiologischer Sicht ist Migration bzw. ein Migrationshintergrund demnach ein wichtiger Faktor für die Beschreibung der gesundheitlichen Ungleichheit einer Bevölkerung. Der Zusammenhang zwischen Migration und Gesundheit ist dabei multidimensional, und der Migrationshintergrund fungiert als eine Art Surrogat für eine Vielzahl von dahinter stehenden Mechanismen und Prozessen. Ohne Einbeziehung des sozialen Status ist die Analyse der gesundheitlichen Situation von Migranten von nur begrenztem Aussagewert. Migranten sollten deshalb mit in sozialepidemiologische Analysen einbezogen und ihre spezifischen Lebensumstände berücksichtigt werden. Oftmals wird dies noch durch eine unzureichende Datenlage erschwert, da in vielen Studien Menschen mit Migrationshintergrund nicht entsprechend ihrem Anteil an der Bevölkerung beteiligt werden. Andererseits ist festzustellen, dass die vorhandenen Daten, die z. B. durch das SOEP oder den Mikrozensus bereitgestellt werden, zu selten genutzt werden. Darüber hinaus ist mit Blick auf die zunehmende Anzahl an Flüchtlingen, die zu einem Großteil langfristig in Deutschland bleiben werden und spezifische Gesundheitsprobleme und Versorgungsbedarfe haben, festzustellen, dass erhebliche Informationsdefizite bestehen. Um diese abzubauen, müssen spezifische Zugänge der Datengewinnung gefunden werden, unter Berücksichtigung von sprachlichen und kulturellen Barrieren sowie der sozialen und gesundheitlichen Situation dieser Bevölkerungsgruppe.

\section{Soziale Unterschiede in der Gesundheitsversorgung}

In den oben aufgeführten Erklärungsmodellen zur gesundheitlichen Ungleichheit wird die gesundheitliche Versorgung als eine Einflussgröße auf die Entstehung - und im günstigen Fall den Abbau - von sozial differenziellen Erkrankungsrisiken angesprochen. Die Frage, ob alle sozioökonomischen Gruppen eine adäquate Gesundheitsversorgung erhalten, muss mit den Methoden der Versorgungsforschung beantwortet werden, die sich mit der gesamten Gesundheitsversorgung inklusive Prävention, Gesundheitsförderung und Krankenversorgung befasst [87].

Die Versorgungsforschung hat unter anderem zeigen können, dass Maßnahmen zur Prävention und Gesundheitsförderung insbesondere von denjenigen Bevölkerungsgruppen in Anspruch genommen werden, die ohnehin einen gesunden Lebensstil und ein geringes Krankheitsrisiko aufweisen. Menschen in sozioökonomisch benachteiligten Positionen hingegen nehmen präventive und gesundheitsfördernde Angebote seltener wahr. Dieses „Präventionsdilemma“ ist beispielsweise aus Studien zur Inanspruchnahme der Schwangerenvorsorge, zur Partizipation an den U1bis U9-Untersuchungen, zu Rückenschulen, zu Maßnahmen zur Tabakentwöhnung und Gewichtsreduktion, zur Zahnprophylaxe und zu Krebsvorsorgeuntersuchungen bekannt [88]. Trotz zielgruppengerechter Bemühungen und partieller Erfolge ist dieses ,preaching to the converted“" [89] im Feld der Prävention 
und Gesundheitsförderung weiterhin ein strukturelles Problem, welches die ohnehin schon bestehende gesundheitliche Ungleichheit noch verstärken kann.

Weniger eindeutig ist die Datenlage im Bereich der Krankenversorgung. Eine methodische Herausforderung ist die nötige Adjustierung nach der tatsächlichen Morbidität der Betroffenen. Schließlich lassen sich Vergleiche zu Inanspruchnahme und Qualität der Krankenversorgung zwischen Bevölkerungsgruppen nur auf Basis ähnlich schwerer Fälle treffen. Daten aus Deutschland weisen auf Status-, Geschlechts- und Migrationseinflüsse in der Inanspruchnahme und im Behandlungserfolg kurativer Maßnahmen hin [90], beispielsweise dergestalt, dass ambulante und stationäre Behandlungsdauer ebenso wie der Behandlungserfolg bei sozial Benachteiligten ungünstiger sind [90]. Darüber hinaus hat die Versorgungsforschung Hinweise darauf geliefert, dass die Einführung der Praxisgebühr [91], die Bevorzugung von Privatpatienten gegenüber Kassenpatienten [92, 93], der bessere Zugang höher Gebildeter zu Fachärzten [94] und eine regionale ambulante Unterversorgung gesundheitliche Ungleichheiten auf struktureller Ebene [95] verstärkt haben könnten [96].

Die Hinwendung zum Gesundheitssystem ist ein relativ neuer Trend in der Forschung zur gesundheitlichen Ungleichheit. Allerdings wird die Bedeutung dieser Forschungsperspektive zunehmend klarer. Zum einen sind die Akteure der Versorgung wichtige Partner beim Abbau gesundheitlicher Ungleichheit, da sie aktiv dazu beitragen können, Versorgungsstrukturen zu verändern und die medizinischen Folgen sozial ungleicher Belastungen zu verringern. Zum anderen profitiert das Gesundheitssystem insgesamt, wenn es gelingt, eine passgenaue Versorgung anzubieten und damit die Wirksamkeit von Maßnahmen zu steigern.

\section{Regionale und sozialräumliche Betrachtung der gesundheitlichen Ungleichheit}

Das räumliche Zusammenfallen von sozialer Benachteiligung und ein- geschränkter Gesundheit ist ein häufig beobachtetes Phänomen, das die Forschung zur sozialen Ungleichheit seit Längerem bewegt. In Deutschland ist beispielsweise die Lebenserwartung in solchen Regionen niedriger, die auch ein niedrigeres Einkommensniveau oder hohe Arbeitslosenraten haben $[18,97]$. In einem Bericht zur gesundheitlichen Lage in Ost- und Westdeutschland 20 Jahre nach dem Mauerfall berichtet das Robert Koch-Institut, dass sozioökonomische Unterschiede mittlerweile viel prägender für die Gesundheit einer Region sind als Ost-West-Unterschiede [98]. Dieser Befund ist nicht nur für die allgemeine Lebenserwartung gültig, sondern auch für einzelne Erkrankungen wie ischämische Herzkrankheiten [99]. Solche räumlichen Ungleichheiten lassen sich zudem bis hinunter auf die Ebene von einzelnen Nachbarschaften in Städten beobachten [100].

Für diese Korrelationen auf ökologischer Ebene bietet sich eine einfache Erklärung an. In Gebieten in denen viele ärmere oder sozioökonomisch benachteiligte Menschen leben, sind die Erkrankungsraten notwendigerweise höher, weil diese Menschen eben aufgrund ihrer individuellen Situation höhere Risiken haben. Solche „kompositionellen Effekte“ reichen aber zur alleinigen Erklärung nicht aus. Das zeigen Studien, die mithilfe von Mehrebenenanalysen einen eigenständigen Effekt von sozialer und ökonomischer Benachteiligung auf räumlicher Ebene auf die individuelle Gesundheit der Bewohner dieser Regionen gefunden haben [101]. Dieser Einfluss vermittelt sich über Faktoren, die in direktem Zusammenhang mit dem Wohlstand einer Gegend stehen. Gemeint sind Faktoren wie beispielsweise die allgemeine Wohnqualität im Wohnviertel, das Verkehrsaufkommen, Kriminalitätsraten, Erholungsmöglichkeiten oder Sportstätten, die in ihrer räumlichen Verteilung oft von ökonomischen und politischen Bedingungen abhängen. Studien belegen, dass solche Umgebungsbelastungen tatsächlich häufiger in „ärmeren “ Gegenden (Stadteile oder Regionen) zu beobachten sind $[102,103]$. Hinzu kommen Einflüsse auf das individuelle Verhalten. Für zahlreiche gesundheitsrelevante Verhaltensweisen, wie z. B. die körperliche Be- wegung, spielen Umgebungseinflüsse eine wichtige Rolle. So fällt es Menschen in Gegenden mit vielen Sportstätten und attraktiven Außenflächen leichter, Sport zu treiben, als Menschen in innerstädtischen Ballungsräumen.

Vor diesem Hintergrund kommt der Diskussion über die finanzielle Lage von Kommunen und um die immer stärkere soziale Segregation in Großstädten auch für die Sozialepidemiologie große Bedeutung zu. Mittlerweile besteht zunehmend die Möglichkeit, in Studien erhobene Individualdaten mit sozialräumlichen Indikatoren zusammenzuspielen. Die vorliegenden Untersuchungen, die sich dies zunutze gemacht haben, machen deutlich, dass Mehrebenenbetrachtungen in erheblichem Maße zum Verständnis gesundheitlicher Ungleichheit beitragen können.

\section{Gesundheitliche Ungleichheit im internationalen Vergleich}

Wie Menschen leben und arbeiten, wie das Einkommen in der Gesellschaft verteilt und wie das Gesundheitssystem organisiert ist: All diese Faktoren entstehen nicht in einem Vakuum [104-106]. Die Commission on Social Determinants of Health hat in ihrem Bericht für die WHO deutlich unterstrichen, dass die Qualität der sozialen Determinanten der Gesundheit durch politische, ökonomische und soziale Kräfte geformt wird, die sich je nach Land und Region unterscheiden können [9]. Verändert man also den Blickwinkel und nimmt eine Makroperspektive ein, die ganze Gesellschaften fokussiert, wird deutlich, dass nicht nur individuelle Merkmale wie soziale und materielle Lebensbedingungen, sondern auch der Wohlstand eines Landes, das Ausmaß der Einkommensungleichheit, der Aufbau des Wohlfahrtsstaates oder die politischen Partizipationsmöglichkeiten Einfluss auf die Gesundheit und das Ausmaß gesundheitlicher Ungleichheit in einer Bevölkerung nehmen [107109].

Während der Zusammenhang zwischen gesellschaftlichem Wohlstand und Gesundheit auf Länderebene gut dokumentiert ist [110], zeigen aktuelle Ergebnisse, dass nicht nur die Höhe des 
Wohlstands, sondern auch seine Verteilung innerhalb der Gesellschaft (z. B. Einkommensungleichheit) eine Rolle für die Gesundheit auf Bevölkerungsebene spielt: Je größer die Kluft zwischen den reichen und armen Bevölkerungsgruppen ist, desto höher sind die gesundheitlichen Beeinträchtigungen. Wie eine Analyse von Wilkinson und Pickett [111] zeigt, lässt sich diese Assoziation für zahlreiche Gesundheitsindikatoren, wie z. B. Säuglingssterblichkeit, subjektive Gesundheit, psychische Störungen, Abhängigkeit von Alkohol und illegalen Drogen, Übergewicht und Adipositas, verfrühte Schwangerschaften bei Teenagern und Gewalt, nachweisen.

Mit der systematischen Überprüfung des Zusammenhangs zwischen Gesundheit, gesundheitlicher Ungleichheit und wohlfahrtsstaatlicher Politik beschäftigten sich jüngere Forschungsarbeiten in ländervergleichender Perspektive [112114]. Aufgrund einer Vielzahl sozialer Leistungen, wie z. B. im Falle von Arbeitslosigkeit, Krankheits- und Arbeitsunfähigkeit und sogenannten Schlüsselleistungen (wie für das Gesundheitssystem oder für soziale Dienste), messen diese Studien der jeweiligen wohlfahrtspolitischen Ausgestaltung einen hohen Stellenwert bei [107]. In einer ländervergleichenden Studie zu Einkommensunterschieden und aufgewendeten Sozialleistungen konnte beispielsweise gezeigt werden, dass Länder, die eine egalitäre Wirtschafts- und Sozialpolitik verfolgten, deutlich niedrigere Säuglings-, Kinderund Gesamtmortalitätsraten aufweisen als weniger egalitäre Länder [115]. Vergleichbare Ergebnisse liegen inzwischen auch für das Jugendalter vor $[116,117]$.

Nicht nur die allgemeine gesundheitliche Lage, sondern auch das Ausmaß sozioökonomischer Disparitäten in der Gesundheit unterscheidet sich nach dem Wohlfahrtsregime. Interessanterweise finden sich in den skandinavischen Wohlfahrtsstaaten nicht die niedrigsten sozioökonomischen Unterschiede in der Gesundheit [106, 113, 114]. Vielmehr weist das konservative Regime oftmals die niedrigsten, das liberale und osteuropäische Regime dagegen die höchsten sozioökonomischen Unterschiede in der Gesundheit auf. Was Ursache und was
Wirkung ist, lässt sich aus solchen Querschnitterhebungen nicht ablesen, und die Forschung steht hier noch am Anfang. Jedoch zeigen die Auswertungen den Wert der Makroperspektive, da diese Informationen unmittelbare Bedeutung für die politische Planung haben können.

\section{Zusammenfassung und Diskussion}

Die Sozialepidemiologie hat sich, ausgehend von ersten Forschungsarbeiten in den 1980er- und frühen 1990er-Jahren, in den letzten zwei Jahrzehnten auch in Deutschland als eigenständige Forschungsrichtung innerhalb der Public-Health- und der epidemiologischen Forschung etabliert. Auch wenn der Anschluss an die Länder, in denen Sozialepidemiologie eine längere Tradition hat, noch nicht ganz geschafft ist - zu verweisen ist unter anderem auf die britischen Geburtskohortenstudien oder die skandinavischen Registerdaten - gibt es in Deutschland inzwischen eine umfassende Datenlage. Die von Jahr zu Jahr zunehmende Anzahl an Studien weist auf einen ausgeprägten und über die Zeit überdauernden Zusammenhang zwischen dem sozialen Status und der Gesundheit bzw. Lebenserwartung hin. Bisweilen wird mit Blick auf den empirischen Forschungsstand sogar festgestellt, dass neue Studien, die sich mit sozialen Unterschieden in der Morbidität und Mortalität befassen, nur von begrenztem Wert sind, da sie lediglich einen inzwischen hinlänglich bekannten $\mathrm{Zu}$ sammenhang belegen. Dagegen ist einzuwenden, dass die regelmäßig wiederholte Analyse der gesundheitlichen Ungleichheit wichtige Hinweise darauf liefert, ob und wie sich die beschriebenen Problemlagen und Verteilungsungleichheiten unter sich wandelnden gesellschaftlichen Verhältnissen reproduzieren. Die kontinuierliche Beschreibung der gesundheitlichen Ungleichheit anhand aktueller Daten und die daran ansetzende Analyse zeitlicher Entwicklungen und Trends sind deshalb als wichtige, dauerhafte Aufgaben der sozialepidemiologischen Forschung anzusehen.

Daneben stellt die Erklärung der beobachteten sozialen Unterschiede in der
Morbidität und Mortalität eine Herausforderung für die sozialepidemiologische Forschung dar. Die vorgestellten Modelle und Schemata dienen zur Systematisierung des bisherigen empirischen Forschungsstands und zeigen Anhaltspunkte für die Erklärung gesundheitlicher Ungleichheit auf. Sie stellen keine Erklärungsmodelle im engeren Sinne dar und lassen sich auch nicht umfassend empirisch überprüfen. Trotz der zum Teil überzeugend beschriebenen Beziehungen zwischen den gesellschaftlichen Rahmenbedingungen, dem sozialen Status und den mit diesem verknüpften Lebensbedingungen und Teilhabechancen, dem Verhältnis von Belastungen und Ressourcen, dem Gesundheitsverhalten und assoziierten Risiken, der gesundheitsbezogenen Versorgung sowie der Gesundheit bzw. Lebenserwartung ergeben sich noch viele offene Fragen. Dies gilt schon deshalb, weil die allgemein formulierten Zusammenhänge zwischen den Faktoren je nach betrachtetem Ungleichheitsindikator und Gesundheitsoutcome und auch der untersuchten Population spezifiziert werden müssen.

Vor dem Hintergrund dieser Überlegungen ergibt sich eine Reihe inhaltlicher Herausforderungen für die sozialepidemiologische Forschung. Dazu zählt, wie bereits erwähnt, die Analyse zeitlicher Entwicklungen und Trends, die durch eine zunehmend bessere Datenlage unterstützt wird. Ebenso stellen lebensphasenspezifische Betrachtungen der gesundheitlichen Ungleichheit eine große Herausforderung dar. Lange Zeit galt das Interesse der sozialepidemiologischen Forschung vorwiegend dem mittleren Lebensalter, weil die Arbeitswelt als zentraler Entstehungsort der Produktion und Reproduktion der gesundheitlichen Ungleichheit angesehen wurde. In den letzten 15 Jahren hat sich das Forschungsinteresse auf das Kindes- und Jugendalter ausgeweitet. Damit wurde dem Umstand Rechnung getragen, dass sich der Einfluss des sozialen Status bereits sehr früh abzeichnet und die gesundheitliche Entwicklung im weiteren Leben nachhaltig prägt. Bislang vergleichsweise selten wird die gesundheitliche Ungleichheit im höheren Lebensalter betrachtet. Vor dem Hintergrund des demo- 
grafischen Wandels rückt dieser Lebensabschnitt aber zunehmend ins Blickfeld. Mittlerweile liegen mehrere für weitere Forschung anschlussfähige Analysen vor, die verdeutlichen, dass sich auch im höheren Alter zum Teil deutliche soziale Unterschiede in der Gesundheit abzeichnen $[118,119]$.

Neben alters- bzw. lebensphasenbezogenen Analysen stellen geschlechterspezifische und -vergleichende Betrachtungen eine bedeutende Perspektive der Sozialepidemiologie dar [120]. Die lange Zeit unterstellte Annahme, dass die gesundheitliche Ungleichheit bei Frauen schwächer ausgeprägt ist als bei Männern, kann inzwischen als widerlegt angesehen werden. In einzelnen Bereichen sind die sozialen Unterschiede bei Frauen sogar stärker ausgeprägt als bei Männern, so z. B. in der Verbreitung der Adipositas. Zum Teil stellen sich die Zusammenhänge auch umgekehrt dar. Ein Beispiel ist der Alkoholkonsum, der bei Frauen anders als bei Männern in der niedrigen Statusgruppe geringer ist als in der hohen Statusgruppe. Die vorliegenden Forschungsergebnisse deuten zudem darauf hin, dass die Erklärung der gesundheitlichen Ungleichheit bei Männern und Frauen unterschiedlich erfolgen muss [121].

Darüber hinaus sind die im vorliegenden Beitrag ausführlich beschriebenen Perspektiven als wichtige Herausforderungen für die sozialepidemiologische Forschung zu verstehen, und zwar, wie bei den vorgenannten, sowohl hinsichtlich der Beschreibung als auch der Erklärung der gesundheitlichen Ungleichheit. Bei der Lebenslaufforschung, der Analyse epigenetischer Mechanismen, migrantenspezifischen Betrachtungen, regionalen und sozialräumlichen Analysen sowie internationalen Vergleichen handelt es sich allesamt um zukunftsträchtige Public-Health-Themen. Die Weiterentwicklung der Sozialepidemiologie und deren Bedeutung innerhalb der Public-Health- und der epidemiologischen Forschung werden entscheidend davon abhängen, inwieweit es gelingt, diese Themen zu integrieren und für die Analyse der gesundheitlichen Ungleichheit zu nutzen.

\section{Korrespondenzadresse}

PD Dr. T. Lampert

Fachgebiet 28, Soziale Determinanten der Gesundheit,

Robert Koch-Institut

General-Pape-Straße 62-66, 12101 Berlin

t.lampert@rki.de

\section{Einhaltung ethischer Richtlinien}

Interessenkonflikt. T. Lampert, M. Richter, S. Schneider, J. Spallek und N. Dragano geben an, dass kein Interessenkonflikt besteht.

Dieser Beitrag beinhaltet keine Studien an Menschen oder Tieren.

\section{Literatur}

1. Mielck A (2000) Soziale Ungleichheit und Gesundheit. Empirische Ergebnisse, Erklärungsansätze, Interventionsmöglichkeiten. Hans Huber, Bern

2. Mielck A (2005) Soziale Ungleichheit und Gesundheit. Einführung in die aktuelle Diskussion. Hans Huber, Bern

3. Richter M, Hurrelmann K (Hrsg) (2009) Gesundheitliche Ungleichheit Grundlagen, Probleme, Perspektiven, 2. aktualisierte Auflage. VS Verlag für Sozialwissenschaften, Wiesbaden

4. Lampert T, Kroll LE, Kuntz B, Ziese T (2011) Gesundheitliche Ungleichheit. In: Statistisches Bundesamt, Wissenschaftszentrum Berlin für Sozialforschung/Zentrales Datenmanagement (Hrsg) Datenreport 2011. Ein Sozialbericht für Deutschland. Bundeszentrale für politische Bildung, Bonn, S 247-258

5. Huster S (2011) Soziale Gesundheitsgerechtigkeit. Sparen, Umverteilen, Versorgen? Wagenbach, Berlin

6. Mielck A, Bloomfield K (Hrsg) (2001) Sozial-Epidemiologie. Eine Einführung in die Grundlagen, Ergebnisse und Umsetzungsmöglichkeiten. Juventa, Weinheims

7. Berkman LF, Kawachi I (Hrsg) (2000) Social epidemiology. Oxford University Press, New York

8. World Health Organization (2013) Health 2020. A European policy framework and strategy for the 21st century. http://www.euro.who.int/en/ publications/abstracts/health-2020-a-europeanpolicy-framework-and-strategy-for-the-21stcentury. Zugegriffen: 17. Juli 2015

9. Commission on Social Determinants of Health (2008) Closing the gap in a generation. Health equity through action on the social determinants of health. Final report of the Commission on Social Determinants of Health. WHO, Geneva

10. Mielck A (1994) „SOziale Medizin“. Die Diskussion zu Beginn des 20. Jahrhunderts. In: Mielck A (Hrsg) Krankheit und soziale Ungleichheit. Sozialepidemiologische Forschungen in Deutschland. Leske + Budrich, Opladen, S 35-52

11. Townsend P, Davidson N, Whitehead M (1990) Inequalities in health: the Black Report and the health divide. Penguin Books Whitehead, London

12. Whitehead M (1987) The health divide. Inequalities in health in the 1980s. Health Education Authority, London
13. Mielck A (1994) Krankheit und soziale Ungleichheit. Sozialepidemiologische Forschungen in Deutschland. Leske + Budrich, Opladen

14. Helmert U, Bammann K, Voges W, Müller R (2001) Müssen Arme früher sterben? Soziale Ungleichheit und Gesundheit in Deutschland. Juventa, Weinheim

15. Bauer U, Bittlingmayer UH, Richter M (Hrsg) (2008) Health inequalities. Determinanten und Mechanismen gesundheitlicher Ungleichheit. VS Verlag für Sozialwissenschaften, Wiesbaden

16. Lampert T, Saß A-C, Häfelinger M, Ziese T (2005) Armut, soziale Ungleichheit und Gesundheit. Expertise zum 2. Armuts- und Reichtumsbericht der Bundesregierung. Beiträge zur Gesundheitsberichterstattung des Bundes. Robert KochInstitut, Berlin

17. Lampert T, Hagen C, Heizmann B (2010) Gesundheitliche Ungleichheit bei Kindern und Jugendlichen in Deutschland. Beiträge zur Gesundheitsberichterstattung des Bundes. Robert KochInstitut, Berlin

18. Lampert T, Kroll LE (2014) Soziale Unterschiede in der Mortalität und Lebenserwartung. GBE kompakt 5/2. Robert Koch-Institut, Berlin

19. Lampert T, Kuntz B, KiGGS Study Group (2015) Gesund aufwachsen - Welche Bedeutung kommt dem sozialen Status zu? GBE kompakt $6 / 1$. Robert Koch-Institut, Berlin

20. Bundesministerium für Arbeit und Soziales (2008) Lebenslagen in Deutschland. Der dritte Armuts- und Reichtumsbericht der Bundesregierung. BMAS, Berlin

21. Bundesministerium für Arbeit und Soziales (2012) Lebenslagen in Deutschland. Der vierte Armuts- und Reichtumsbericht der Bundesregierung. BMAS, Berlin

22. Sachverständigenrat zur Begutachtung der Entwicklung im Gesundheitswesen (2008) Sondergutachten 2007: Kooperation und Verantwortung - Voraussetzungen einer zielorientierten Gesundheitsversorgung. Nomos, Baden-Baden

23. Sachverständigenrat zur Begutachtung der Entwicklung im Gesundheitswesen (2010) Sondergutachten 2009: Koordination und Integration-Gesundheitsversorgung in einer Gesellschaft des längeren Lebens. Nomos, Baden-Baden

24. Busch MA, Schienkiewitz A, Nowossadeck $E$, Gößwald A (2013) Prävalenz des Schlaganfalls bei Erwachsenen im Alter von 40 bis 79 Jahren in Deutschland. Ergebnisse der Studie zur Gesundheit Erwachsener in Deutschland (DEGS1). Bundesgesundheitsbl Gesundheitsforsch Gesundheitsschutz 56:656-660

25. Gößwald A, Schienkiewitz A, Nowossadeck E, Busch MA (2013) Prävalenz von Herzinfarkt und koronarer Herzkrankheit bei Erwachsenen im Alter von 40 bis 79 Jahren in Deutschland. Ergebnisse der Studie zur Gesundheit Erwachsener in Deutschland (DEGS1). Bundesgesundheitsbl Gesundheitsforsch Gesundheitsschutz 56:650655

26. Lampert $\mathrm{T}$, Kroll LE, von der Lippe $\mathrm{E}$, Müters S, Stolzenberg H (2013) Sozioökonomischer Status und Gesundheit. Ergebnisse der Studie zur Gesundheit Erwachsener in Deutschland (DEGS1). Bundesgesundheitsbl Gesundheitsforsch Gesundheitsschutz 56:814821

27. Peter R, Geyer S (1999) Schul- und Berufsausbildung, Berufsstatus und Herzinfarkt - Eine Studie mit Daten einer gesetzlichen Krankenversicherung. Gesundheitswesen 61(1):20-26 
28. Geyer S (2008) Sozialstruktur und Krankheit. Analysen mit Daten der Gesetzlichen Krankenversicherung. Bundesgesundheitsbl Gesundheitsforsch Gesundheitsschutz 51(10):1164-1172

29. Geyer S (2008) Social inequalities in the incidence and case fatality of cancers of the lung, the stomach, the bowels, and the breast. Cancer Causes Control 19(9):965-974

30. Busch MA, Maske U, Ryl L, Hapke U (2013) Prävalenz von depressiven Symptomen und diagnostizierter Depression bei Erwachsenen in Deutschland. Ergebnisse der Studie zur Gesundheit Erwachsener in Deutschland (DEGS1). Bundesgesundheitsbl Gesundheitsforsch Gesundheitsschutz 56:733-739

31. Green MJ, Benzeval M (2011) Ageing, social class and common mental disorders: longitudinal evidence from three cohorts in the West of Scotland. Psychol Med 41:565-574

32. Langen U, Schmitz R, Steppuhn H (2013) Häufigkeit allergischer Erkrankungen in Deutschland. Ergebnisse der Studie zur Gesundheit Erwachsener in Deutschland (DEGS1) Bundesgesundheitsbl Gesundheitsforsch Gesundheitsschutz 56:698-706

33. Haftenberger M, Laußmann D, Ellert U, Kalcklösch M, Langen U, Schlaud M, Schmitz R, Thamm M (2013) Prävalenz von Sensibilisierungen gegen Inhalationsund Nahrungsmittelallergene. Ergebnisse der Studie zur Gesundheit Erwachsener in Deutschland (DEGS1). Bundesgesundheitsbl Gesundheitsforsch Gesundheitsschutz 56:687697

34. Dragano N, Lampert T, Siegrist J (2010) Wie baut sich soziale und gesundheitliche Ungleichheit im Lebenslauf auf? In: Deutsches Jugendinstitut (Hrsg) Mehr Chancen für gesundes Aufwachsen. Materialien zum 13. Kinder- und Jugendbericht. Verlag Deutsches Jugendinstitut, München, S 13-50

35. Müters S, Lampert T, Maschewsky-Schneider U (2005) Subjektive Gesundheit als Prädiktor für Mortalität. Gesundheitswesen 67(3):129-136

36. Babitsch B, Lampert T, Müters S, Morfeld M (2009) Ungleiche Gesundheitschancen bei Erwachsenen: Zusammenhänge und mögliche Erklärungsansätze. In: Richter M, Hurrelmann K (Hrsg) Gesundheitliche Ungleichheit. Grundlagen, Probleme, Perspektiven, 2. aktualisierte Auflage. VS Verlag für Sozialwissenschaften, Wiesbaden, S 231-252

37. Lampert $T$, von der Lippe $E$, Müters S (2013) Verbreitung des Rauchens in der Erwachsenenbevölkerung in Deutschland. Ergebnisse der Studie zur Gesundheit Erwachsener in Deutschland (DEGS1). Bundesgesundheitsbl Gesundheitsforsch Gesundheitsschutz 56:802808

38. Lampert T (2013) Soziale Ungleichheit und Gesundheit. Die sozial ungleiche Verteilung der Erkrankungsrisiken und Gesundheitschancen in Deutschland. Medizinische Fakultät der Universität Leipzig, Leipzig. (Habilitationsschrift)

39. Hoebel J, Finger J, Kuntz B, Lampert T (2016) Sozioökonomische Unterschiede in der körperlich-sportlichen Aktivität von Erwerbstätigen im mittleren Lebensalter:Welche Rolle spielen Bildung, Beruf und Einkommen? doi: 10.1007/ s00103-015-2278-3
40. Finger J, Tylleskär T, Lampert T, Mensink GBM (2012) Physical activity patterns and socioeconomic position: the German National Health Interview and Examination Survey 1998 (GNHIES). BMC Public Health 12:1079

41. Schneider S (2007) Zur diametralen Wirkung körperlicher Bewegung in Beruf und Freizeit auf das Rückenschmerzrisiko - Eine bundesweite Repräsentativstudie unter Berücksichtigung weiterer sozialer Risikofaktoren. Deut Z Sportmed 58:433-440

42. Heindl I (2007) Ernährung, Gesundheit und soziale Ungleichheit. Aus Politik Zeitgeschichte 46:25-31

43. Jordan S, von der Lippe E (2013) Teilnahme an verhaltenspräventiven Maßnahmen. Ergebnisse der Studie zur Gesundheit Erwachsener in Deutschland (DEGS1). Bundesgesundheitsbl Gesundheitsforsch Gesundheitsschutz 56:878884

44. Hoebel J, Richter M, Lampert T (2013) Sozialer Status und Teilnahme am Gesundheits-Checkup von Männern und Frauen in Deutschland: Ergebnisse der GEDA-Studie 2009 und 2010 Deutsches Ärzteblatt 110(41):679-685.

45. von dem Knesebeck O, Bauer U, Geyer S, Mielck A (2009) Soziale Ungleichheit in der gesundheitlichen Versorgung - Ein Plädoyer für systematische Forschung. Gesundheitswesen 71:59-62

46. Reil-Held A (2000) Einkommen und Sterblichkeit in Deutschland: Leben Reiche länger? Sonderforschungsbereich 504, Discussion Paper No. 0014. Universität Mannheim, Mannheim

47. Lampert T, Kroll LE, Dunkelberg A (2007) Soziale Ungleichheit der Lebenserwartung in Deutschland. Aus Politik Zeitgeschichte 42:11-18

48. Lampert T, Richter M (2009) Gesundheitliche Ungleichheit bei Kindern und Jugendlichen. In: Richter M, Hurrelmann K (Hrsg) Gesundheitliche Ungleichheit. Grundlagen, Probleme, Perspektiven. VS Verlag für Sozial wissenschaften, Wiesbaden, S 209-230

49. Lampert $T$ (2010) Frühe Weichenstellung. Zur Bedeutung der Kindheit und Jugend für die Gesundheit im späteren Leben. Bundesgesundheitsbl Gesundheitsforsch Gesundheitsschutz 53(5):486-497

50. Schneider S, Maul H, Freerksen N, PötschkeLanger M (2008) Who smokes during pregnancy? - Analysis of the "German Perinatal Quality Survey 2005". Public Health 122:1210-1216

51. Dragano N, Siegrist J (2009) Die Lebenslaufperspektive gesundheitlicher Ungleichheit: Konzepte und Forschungsergebnisse. In: Richter M, Hurrelmann K (Hrsg) Gesundheitliche Ungleichheit. Grundlagen, Probleme, Perspektiven, 2. aktualisierte Auflage. VS Verlag für Sozialwissenschaften, Wiesbaden, S 181-194

52. World Health Organization (2003) Social determinants of health. The solid facts, 2. Aufl. WHO Regional Office for Europe, Copenhagen

53. Marmot M (2004) The status syndrome. How social standing affects our health and longevity. Times Books, New York

54. Becker R (1998) Bildung und Lebenserwartung in Deutschland. Z Soziol 27(2):133-150

55. Elkeles T, Mielck A (1997) Entwicklung eines Modells zur Erklärung gesundheitlicher Ungleichheit. Gesundheitswesen 59(3):137-143
56. Bolte $G$, Bunge $C$, Hornberg C, Köckler H, Mielck A (2012) Umweltgerechtigkeit. Chancengleichheit bei Umwelt und Gesundheit: Konzepte, Datenlage und Handlungsperspektiven. Hans Huber, Bern

57. Siegrist J, Theorell T (2006) Socio-economic position and health: the role of work and employment. In: Social inequalities in health. Oxford University Press, Oxford, S 73-100

58. Gee GC, Payne-Sturges DC (2004) Environmental Health Disparities: a framework integrating psychosocial and environmental concepts. Environ Health Perspect 112(17):1645-1653

59. Janßen C, Swart E, von Lengerke T (2013) Theorizing, empiricizing, and analyzing health care utilization in germany: an introduction. In: Janßen C, Swart E, von Lengerke T (Hrsg) Health care utilization in Germany. theory, methodology, and results. Springer, New York, S 3-10

60. Klein J, Hofreuter-Gätgens K, von dem Knesebeck O (2014) Socioeconomic status and the utilization of health services in Germany: a systematic review. In: Janßen C, Swart E, von Lengerke T (Hrsg) Health care utilization in Germany: theory, methodology and results. Springer, New York, S 117-143

61. Hradil S (2009) Was prägt das Krankheitsrisiko? Schicht, Lage, Lebensstil? In: Richter M, Hurrelmann K (Hrsg) Gesundheitliche Ungleichheit. Grundlagen, Probleme, Perspektiven, 2. aktualisierte Auflage. VS Verlag für Sozialwissenschaften, Wiesbaden, S 35-54

62. Commission on Social Determinants of Health (2007) A conceptual framework for action on the social determinants of health. Discussion paper for the Commission on Social Determinants of Health. WHO, Copenhagen

63. Pons-Vigués M, Diez È, Morrison J, Salas-Nicás S, Hoffmann R, Burstrom B, van Dijk JP, Borrell C (2014) Social and health policies or interventions to tackle health inequalities in European cities: a scoping review. BMC Public Health 14(1):198

64. Lynch J, Davey Smith G (2005) A life course approach to chronic disease epidemiology. Annu Rev Public Health 26:1-35

65. Barker DJP (1998) Mothers, babies and health in later life, 2. Aufl. Churchill Livingstone, Edinburgh

66. Kuh D, Ben-Shlomo Y (Hrsg) (2005) A life course approach to chronic disease. Epidemiology, 2. Aufl. Oxford University Press, Oxford

67. Graham H (2002) Building an inter-disciplinary science of health inequalities: the example of life course research. Soc Sci Med 55:2005-2016

68. Gillman MW (2004) A life course approach to obesity. In: Kuh D, Ben Shlome Y (Hrsg) A life course approach to chronic disease epidemiology. Oxford University Press, Oxford, S 189-217

69. Malina RM (1996) Tracking of physical activity and physical fitness across the life span. Res $Q$ Exerc Sport 67(Supplement 3):S48-S57

70. Quenzel G, Hurrelmann K (2010) Bildungsverlierer. Neue Ungleichheiten. VS Verlag für Sozialwissenschaften, Wiesbaden

71. Dragano N, Wahrendorf M (2014) Consistent health inequalities in Europe: the importance of labour market disadvantages. J Epidemiol Community Health 68:293-294

72. Gianaros PJ, Manuck SB (2010) Neurobiological pathways linking socioeconomic position and health. Psychosom Med 72(5):450-461

73. Cole SW (2009) Social regulation of human gene expression. Curr Dir Psychol Sci 18(3):132-137 
74. Miller G, Chen E, Cole SW (2009) Health psychology: developing biologically plausible models linking the social world and physical health. Annu Rev Psychol 60:501-524

75. Seeman TE, Epel E, Gruenewald T, Karlamangla A, McEwen BS (2010) Socio-economic differentials in peripheral biology: cumulative allostatic load. Ann N Y Acad Sci 1186:223-239

76. Szyf M, McGowan P, Meaney MJ (2008) The social environment and the epigenome. Environ Mol Mutagen 49(1):46-60

77. Shanahan MJ, Hofer SM (2010) Molecular genetics, aging, and the life course: sensitive periods, accumulation, and pathways models. In: Binstock RH, George L (Hrsg) Handbook of aging and the social sciences. Elsevier, New York

78. McGuinness D, McGlynn LM, Johnson PC, MacIntyre A, Batty GD, Burns H, Cavanagh J, Deans KA, Ford I, McConnachie A, McGinty A, McLean JS, Millar K, Packard CJ, Sattar NA, Tannahill C, Velupillai YN, Shiels PG (2012) Socioeconomic status is associated with epigenetic differences in the pSoBid cohort. Int J Epidemiol 41(1):151-160

79. Borghol N, Suderman M, McArdle W et al (2012) Associations with early-life socio-economic position in adult DNA methylation. Int J Epidemiol 41:62-74

80. Needham BL, Fernandez JR, Lin J et al (2012) Socioeconomic status and cell aging in children. Soc Sci Med 74:1948-1951

81. Matthews KA, Gallo LC (2011) Psychological perspectives on pathways linking socioeconomic status and physical health. Annu Rev Psychol 62:501-530

82. Halfon N, Larson K, Lu M, Tullis E, Russ S (2014) Lifecourse health development: past, present and future. Matern Child Health J 18(2):344-365

83. Haggarty P, Ferguson-Smith AC (2014) Life Course Epigenetics and healthy ageing. In: Kuh D, Cooper R, Hardy R, Richards M, Ben-Shlomo Y (Hrsg) A life course approach to healthy ageing. Oxford Univ Pr., Oxford, S 198-214

84. Spallek J, Zeeb H, Razum O (2011) What do we have to know from migrants' past exposures to understand their health status? A life course approach. Emerg Themes Epidemiol 8(6):11

85. Razum, O Zeeb H, Meesmann U, Schenk L, Bredehorst M, Brzoska P, Dercks T, Glodny $S$, Menkhaus B, Salman R, Saß AC, Ulrich R (2008) Migration und Gesundheit. Schwerpunktbericht der Gesundheitsberichterstattung des Bundes. Robert Koch-Institut, Berlin

86. Schott T, Razum $O$ (2013) Migration und medizinische rehabilitation. Beltz-Juventa, Weinheim

87. Pfaff H (2003) Versorgungsforschung - Begriffsbestimmung, Gegenstand und Aufgaben. In: Pfaff H, Schrappe M, Lauterbach KW, Engelmann U, Halber M (Hrsg) Gesundheitsversorgung und Disease Management. Grundlagen und Anwendungen der Versorgungsforschung. Hans Huber, Bern, S13-23

88. Janssen C, Swart E, von Lengerke T (Hrsg) (2014) Health care utilization in Germany: theory, methodology, and results. Springer, Heidelberg

89. Schneider S, Schiltenwolf M (2005) „Preaching to the converted" - Über- und Unterversorgung in der Schmerzprävention am Beispiel bundesdeutscher Rückenschulen. Schmerz 19:477-488
90. Janßen C, Grosse Frie K, Dinger H, Schiffmann L, Ommen $O$ (2009) Der Einfluss von sozialer Ungleichheit auf die medizinische und gesundheitsbezogene Versorgung in Deutschland. In: Richter M, Hurrelmann K (Hrsg) Gesundheitliche Ungleichheit. Grundlagen, Probleme, Perspektiven, 2. Aufl. VS Verlag für Sozialwissenschaften, Wiesbaden, S 149-165

91. Gebhardt B (2005) Zwischen Steuerungswirkung und Sozialverträglichkeit - eine Zwischenbilanz zur Praxisgebühr aus Sicht der Versicherten. In: Böcken J, Braun B, Schnee M, Amhof R (Hrsg) Gesundheitsmonitor 2005. Die ambulante Versorgung aus Sicht von Bevölkerung und Ärzteschaft. Verlag Bertelsmann Stiftung, Gütersloh, S11-31

92. Lungen M, Stollenwerk B, Messner P, Lauterbach KW, Gerber A (2008) Waiting times for elective treatments according to insurance status: a randomized empirical study in Germany. Int J Equity Health 7(1):1-7

93. Becklas E, Mielck A, Böcken J (2012) Das ArztPatienten-Verhältnis in der ambulanten Versorgung - Unterschiede zwischen GKV- und PKVVersicherten. In: Böcken J, Braun B, Repschläger U (Hrsg) Gesundheitsmonitor 2012. Bürgerorientierung im Gesundheitswesen. Kooperationsprojekt der Bertelsmann Stiftung und der BARMER/GEK. Verlag Bertelsmann Stiftung, Gütersloh, S 28-53

94. Stirbu I, Kunst AE, Mielck A, Mackenbach JP (2011) Inequalities in utilization of general practitioner and specialist services in 9 European countries. BMC Health Serv Res 11:288

95. Albrecht M, Etgeton S, Ochmann RR (2014) Faktencheck Gesundheit. Regionale Verteilung von Arztsitzen (Ärztedichte). Haus-, Kinder-, Frauen- und Augenärzte. Verlag Bertelsmann Stiftung, Gütersloh

96. Gerlinger T (2007) Soziale Ungleichheit von Gesundheitschancen: Anmerkungen zum Beitrag der Gesundheitspolitik. Diskussionspapier 2007 - 2. Institut für medizinische Soziologie, Frankfurt a. M.

97. Gaber E, Wildner M (2011) Sterblichkeit, Todesursachen und regionale Unterschiede. Gesundheitsberichterstattung des Bundes, Heft 52. Robert Koch-Institut, Berlin

98. Robert Koch-Institut (2010) 20 Jahre nach dem Mauerfall: Wie hat sich die Gesundheit in Deutschland entwickelt? Beiträge zur Gesundheitsberichterstattung des Bundes. RKI, Berlin

99. Stang A, Stang M (2014) Kardiovaskuläre Risikofaktoren im Bundeslandvergleich. Dtsch Arztebl Int 111:530-536

100. Van Lenthe FJ (2008) Aggregierte Deprivation und ihre Folgen für die Gesundheit. In: Siegrist J, Marmot M (Hrsg) Soziale Ungleichheit und Gesundheit. Erklärungsansätze und gesundheitspolitische Folgerungen. Hans Huber, Bern, S 213243

101. Diez Roux AV (2008) Next steps in understanding the multilevel determinants of health. J Epidemiol Community Health 62:957-959

102. Franzini L, Caughy M, Spears W, Fernandez Esquer ME (2005) Neighborhood economic conditions, social processes, and self-rated health in low-income neighborhoods in Texas: a multilevel latent variables model. Soc Sci Med 61:1135-1150

103. Mohnen S, Schneider S (2014) Wohnumgebung und Gesundheit. In: Bucksch J, Schneider S (Hrsg) Walkability - Bewegungsförderung in der Kommune. Hans Huber, Bern, S 27-44.
104. Raphael D (2006) Social determinants of health: present status, unresolved questions, and future directions. Int J Health Serv 36:651-677

105. Regidor $E$ (2006) Social determinants of health: a veil that hides socioeconomic position and its relation with health. J Epidemiol Community Health 60:896-901

106. Rathmann K (2015) Bildungssystem, Wohlfahrtsstaat und gesundheitliche Ungleichheit. Ein internationaler Vergleich für das Jugendalter. VS Verlag für Sozialwissenschaften, Wiesbaden

107. Borrell C, Espelt A, Rodríguez-Sanz M, Navarro V (2007) Politics and health. J Epidemiol Community Health 61:658-659

108. Hurrelmann K, Rathmann K, Richter M (2011) Health inequalities and welfare state regimes. $A$ research note. J Public Health 19:3-13

109. Mackenbach JP (2012) The persistence of health inequalities in modern welfare states: the explanation of a paradox. Soc Sci Med 75(4):761769

110. Muntaner C, Borrell C, Ng E et al (2011) Review article: politics welfare regimes, and population health: controversies and evidence. Sociol Health IIIn 33:946-964

111. Wilkinson RG, Pickett $K$ (2009) The spirit level. Why more equal societies almost always do better. Bloomsbury Press, London

112. Bambra C (2006) Health status and the worlds of welfare. Social Policy Society 5:53-62

113. Eikemo TA, Bambra C, Joyce K, Dahl E (2008) Welfare state regimes and income-related health inequalities: a comparison of 23 European countries. Eur J Pub Health 18:593-599

114. Eikemo TA, Bambra C, Judge K, Ringdal K (2008) Welfare state regimes and differences in selfperceived health in Europe: a multilevel analysis. Soc Sci Med 66:2281-2295

115. Coburn D (2004) Beyond the income inequality hypothesis: class, neo-liberalism, and health inequalities. Soc Sci Med 58:51-56

116. Richter M, Rathmann K, Nic Gabhainn S, Zambon A, Boyce W, Hurrelmann K (2012) Welfare state regimes, health and health inequalities in adolescence: a comparative study in 35 countries. Sociol Health IIIn 34(6):858-879

117. Rathmann $K$, Ottova V, Hurrelmann $K$, de Looze M, Levin K, Molcho M, Elgar F, Richter M (2015) Macro-level determinants of young people's subjective health and health inequalities: a multilevel analysis in 27 welfare states. Maturitas 80(4):414-420

118. von dem Knesebeck O, Schäfer I (2009) Gesundheitliche Ungleichheit im höheren Lebensalter. In: Richter M, Hurrelmann K (Hrsg) Gesundheitliche Ungleichheit Grundlagen, Probleme, Perspektiven, 2. aktualisierte Auflage. VS Verlag für Sozialwissenschaften, Wiesbaden, S 253-282

119. Huisman M, Kunst AE, Mackenbach JP (2003) Socioeconomic inequalities in morbidity among the elderly: a European overview. Soc Sci Med 57:861-873

120. Bartley M (2004) Health inequality. An introduction to theories, concepts and methods. MPG Books, Bodmin

121. Babitsch $B$ (2005) Soziale Ungleichheit, Geschlecht und Gesundheit. Hans Huber, Bern 